\title{
Embolization of a Mesenteric Arteriovenous Malformation Associated with Idiopathic Myointimal Hyperplasia: A Case Report and Literature Review
}

\author{
Yash B Shah ( yxs049@students.jefferson.edu ) \\ Thomas Jefferson University Sidney Kimmel Medical College https://orcid.org/0000-0003-1551-7611 \\ Derek Lee \\ University of Pennsylvania Department of Radiology \\ Tamim S. Khaddash \\ University of Pennsylvania Department of Radiology
}

\section{Research Article}

Keywords: Embolization, Mesenteric Fistula, IMHMV, Ischemic Colitis, Arteriovenous Malformations, Endovascular Treatment

Posted Date: September 16th, 2021

DOl: https://doi.org/10.21203/rs.3.rs-909156/v1

License: (c) (1) This work is licensed under a Creative Commons Attribution 4.0 International License.

Read Full License 
Manuscript Title: Embolization of a Mesenteric Arteriovenous Malformation Associated with Idiopathic Myointimal Hyperplasia: A Case Report and Literature Review

Running Head: Case Report: AV Malformation Embolization and IMHMV

Authors: Yash B. Shah ${ }^{1}$, Derek Lee ${ }^{2}$, Tamim S. Khaddash ${ }^{2}$

${ }^{1}$ Sidney Kimmel Medical College, Thomas Jefferson University, 1025 Walnut St, Philadelphia, PA, 19107, USA, https://orcid.org/0000-0003-1551-7611

${ }^{2}$ Department of Interventional Radiology, Hospital of the University of Pennsylvania, 3400 Spruce St, Philadelphia, PA, 19104, USA

\section{Corresponding Author:}

Yash B. Shah

Address: 1025 Walnut St, Philadelphia, PA, 19107, USA

Email: yashah01@gmail.com; yxs049@students.jefferson.edu

Phone: 484-365-3809

\section{Abstract Word Count: 230 \\ Text Word Count: 1327 \\ Reference Count: 8 \\ Figure/Table Count: 4}

Keywords: Embolization, Mesenteric Fistula, IMHMV, Ischemic Colitis, Arteriovenous Malformations, Endovascular Treatment

Abbreviations: IMHMV (idiopathic myointimal hyperplasia of the mesenteric vein), IBD (inflammatory bowel disease), IMA (inferior mesenteric artery), AVM (arteriovenous malformation) 


\begin{abstract}
Background: Idiopathic myointimal hyperplasia of the mesenteric vein (IMHMV) is a rare, often undiagnosed pathology affecting the colon. Patients typically present with severe abdominal pain and inflammation caused by smooth muscle proliferation of the veins, leading to arterialization, stenosis, and potential occlusion. The etiology remains unclear, but it has been hypothesized that an arteriovenous connection may be associated with the pathology. This is the first reported case supporting such an association. Endovascular embolization is generally a less invasive alternative to surgical resection in the treatment of such vascular disorders, and our case highlights the potential benefits of endovascular treatment.

Case Presentation: This report describes a 24-year-old female patient with findings of colitis and an abnormal arteriovenous connection of the inferior mesenteric arterial and venous system. Partial embolization of this arteriovenous connection temporarily improved symptoms, but the presence of multiple smaller feeder vessels not amenable to embolization ultimately necessitated colonic resection for definitive treatment. Although prior reports have hypothesized that arterial pressurization of the veins may cause myointimal hyperplasia, to the authors' knowledge, this is the first report of IMHMV with an associated abnormal arteriovenous connection.

Conclusions: This case illustrates the possibility of a pathogenic relationship between an arteriovenous connection and IMHMV. It is presumable that early radiologic analysis for an abnormal arteriovenous connection in colitis patients may facilitate IMHMV diagnosis, and early diagnosis may even facilitate the success of embolization.
\end{abstract}




\section{Background}

Idiopathic myointimal hyperplasia of the mesenteric vein (IMHMV) is an uncommon entity typically affecting the distal and rectosigmoid colon. Its clinical presentation and pathogenesis resemble that of inflammatory bowel disease (IBD); however, biopsy of the affected colon may show findings of ischemia without classic IBD features. Prevalence is likely underreported, as the disease typically remains undiagnosed during the clinical course until histopathology of resected colon is reviewed. Little is known about the etiology and pathogenesis of IMHMV. This report describes the unique clinical progression of a patient with clinical and radiologic findings of colitis in addition to an abnormal arteriovenous connection of the inferior mesenteric artery (IMA) and the venous system. Prior reports have hypothesized that arterial pressurization of the venous vasculature may cause venous myointimal hyperplasia, but to the authors' knowledge, there have been no reports of IMHMV in a patient with angiographic evidence of an abnormal arteriovenous connection.

Partial endovascular embolization of this arteriovenous connection resulted in temporary improvement of the patient's symptoms, but she ultimately required definitive treatment with partial colonic resection. Despite this outcome, endovascular embolization has proven to be a viable alternative to surgical resection and is less invasive in the treatment of vascular disorders such as arteriovenous malformations (AVMs) ${ }^{1}$. We believe that further implementation of endovascular approaches to diagnostic evaluation and treatment for patients presenting with colitis may improve IMHMV detection and treatment.

\section{Case Presentation}

A 24-year-old female with no significant past medical history initially presented to an outside hospital with severe abdominal pain and bloody stools. She was transferred to our tertiary care center for further work-up and management. Contrast-enhanced CT of the abdomen and pelvis showed findings of colitis extending from the splenic flexure to the rectum (Figure 1). The patient was empirically treated for infectious and inflammatory etiologies of colitis without improvement. Flexible sigmoidoscopy demonstrated inflammatory changes in the descending colon and rectum with biopsy samples suggestive of ischemic colitis. Differential diagnosis for possible ischemic colitis included thrombosis and vasculitis, but there were no definitive imaging findings of either on CT imaging. Hematologic and rheumatologic work-up was nonspecific but notable for elevated rheumatoid factor and positive antinuclear antibodies.

Interventional radiology was consulted for further angiographic evaluation of the mesenteric vessels given the ischemic findings on pathology. IMA angiography was performed, and prompt filling of IMA and paralleling mesenteric venous branches was observed (Figure 2). A few abnormal-appearing, tortuous distal IMA branches were noted. Additionally, no IMV was seen on delayed imaging, possibly due to chronic occlusion.

Given these findings, the patient was empirically treated with steroids and anticoagulation. She showed marginal improvement with persistent severe abdominal pain. Based on the prompt venous filling seen on conventional angiography, it was hypothesized that the presence of an arteriovenous connection may be causing mesenteric venous congestion. After a 
multidisciplinary team discussion, it was decided to pursue further angiographic investigation and attempt endovascular embolization of a suspected arteriovenous malformation.

The patient returned to interventional radiology for further diagnostic imaging and potential intervention. The right common femoral artery was accessed using standard micropuncture technique. Digital subtraction angiography of the IMA was performed using a 5 French catheter. Using a microcatheter and microwire, further selective angiography of the IMA branches was performed. Filling of the venous system was again seen along with a tangle of small vessels compatible with a nidus connecting small arterial feeders to the venous system (Figure 3). Glue embolization of this nidus was performed. Post-embolization imaging demonstrated decreased venous filling, however, there was persistent venous filling from several smaller IMA feeding branches, which were small and not amenable to embolization (Figure 4).

The patient's pain significantly improved the day after embolization, and she was discharged 5 days post-embolization. However, her pain returned in 11 days and rose to pre-embolization levels approximately 26 days post-embolization. She was readmitted for pain management, and her deteriorating condition resulted in a multidisciplinary medicine, interventional radiology, and surgery team decision to proceed with colonic resection. Extended left colectomy with partial proctectomy, end transverse colostomy, and rectal stump were performed. The patient tolerated the procedure well, and her pain completely resolved. Pathological analysis of resection specimens indicated prominent myointimal hyperplasia with colonic perforation. Van Gieson stain along with venous presence of lymphocytic phlebitis, fat necrosis, and organizing thrombi confirmed IMHMV diagnosis ${ }^{2}$.

\section{Conclusions}

IMHMV is a rare disorder with only 34 documented cases in the literature. It has classically been reported in otherwise healthy young to middle-aged men, although recent cases, including ours, have identified the disease in females or older patients ${ }^{3}$. Clinical diagnosis of IMHMV is difficult and generally made via histopathology after surgical resection ${ }^{3,4}$. Patients present with nonspecific symptoms including severe abdominal pain, weight loss, rectal bleeding, and diarrhea or constipation that mimic $\mathrm{IBD}^{5}$. Initial radiologic evaluation of IMHMV patients is typically suggestive of colitis, and endoscopic findings show nonspecific inflammatory findings, both of which were seen in our patient.

Like our case, patients typically undergo a protracted clinical course of colitis that ultimately requires surgical resection. Our patient is unique as her colitis was thought to be secondary to an abnormal arteriovenous connection, which was discovered on conventional angiography performed during mesenteric ischemia work-up.

While uncommon, mesenteric arteriovenous abnormalities are well documented, with multiple case reports describing successful endovascular embolization of arteriovenous fistulas and malformations ${ }^{1,6,7}$. Endovascular embolization has proven to be a viable alternative to surgical resection, as it is less invasive and presents fewer complications, although it does carry potential for bowel ischemia. While only partial embolization was achieved in our case, the patient saw significant pain reduction and her opioid requirement decreased in the subsequent days. Since 
partial embolization was performed, we acknowledged the possibility that her pain would return in the future as new arterial feeders develop post-embolization and repressurize the venous system. Additionally, retrospectively upon further evaluation and discussion, we acknowledge that the abnormal arteriovenous connection seen in this patient may not have represented a true AVM, but a secondary, nonspecific arteriovenous connection associated with either IMHMV and/or chronic thrombosis of the IMV.

The definitive etiologies of both mesenteric AVM and IMHMV are unknown. It is likely that AVM and IMHMV are associated, but no previous reports have proven the basis of this association. IMHMV is characterized by smooth muscle proliferation in small and medium-sized mesenteric veins, in essence creating venous arterialization which leads to stenosis and possible occlusion. Thus, prior reports have hypothesized that IMHMV results from an arteriovenous connection that leads to increased pressurization of the veins, resulting in venous arterialization. To our knowledge, this is the first reported case of mesenteric arteriovenous abnormality associated with IMHMV on histopathology.

Standard treatment for both IMHMV and AVM is surgical resection of the affected section of bowel. However, endovascular embolization has also been shown to be a viable treatment option for arteriovenous connections where the anatomy is amenable. While embolization did not yield long-term benefit for our patient, we believe that given an earlier radiologic diagnosis, it could offer a viable alternative to resection. There have not yet been any reports of successful conservative medical treatment for IMHMV and the median time from patient presentation and symptom onset to surgical resection is 5 months. Typically, diagnosis is missed, and surgery is performed after significant morbidity or failure to respond to IBD medical treatments, including corticosteroids, which may result in severe morbidity and mortality ${ }^{8}$.

This case illustrates the significance of an arteriovenous connection in the setting of ischemic colitis, with a plausible pathogenic relationship to IMHMV. Coupling the clinical presentation of this patient with the previously hypothesized associations with arteriovenous malformations, it is presumable that early radiologic analysis for an abnormal arteriovenous connection may facilitate IMHMV diagnosis. Earlier diagnosis may also facilitate increased success of endovascular treatment, which offers benefits compared to surgical resection ${ }^{6}$. With increased awareness of the condition, IMHMV can be considered as a potential diagnosis in patients presenting with protracted severe abdominal pain and colitis without a clear infectious or inflammatory etiology. 


\section{References}

1. Hussein M, Issa G, Muhsen S, Haydar A. Superior mesenteric arteriovenous fistula embolisation complicated by bowel ischaemia. BMJ Case Rep. May 15

2013;2013doi:10.1136/bcr-2013-009521

2. Genta RM, Haggitt RC. Idiopathic myointimal hyperplasia of mesenteric veins.

Gastroenterology. Aug 1991;101(2):533-9. doi:10.1016/0016-5085(91)90035-j

3. Platz J, Hyman N. Idiopathic myointimal hyperplasia of mesenteric veins. Gastroenterol Hepatol (N Y). Oct 2012;8(10):700-2.

4. Yun SJ, Nam DH, Kim J, Ryu JK, Lee SH. The radiologic diagnosis of idiopathic myointimal hyperplasia of mesenteric veins with a novel presentation: case report and literature review. Clin Imaging. Sep-Oct 2016;40(5):870-4. doi:10.1016/j.clinimag.2015.12.017

5. Song SJ, Shroff SG. Idiopathic Myointimal Hyperplasia of Mesenteric Veins of the Ileum and Colon in a Patient with Crohn's Disease: A Case Report and Brief Review of the Literature. Case Rep Pathol. 2017;2017:6793031. doi:10.1155/2017/6793031

6. Hendy P, Cheng EH, Livsey R, Mortimore M. Curative embolization of an inferior mesenteric arteriovenous fistula causing ischaemic proctosigmoiditis. ANZ J Surg. Apr 2018;88(4):E340-E341. doi:10.1111/ans.13346

7. Athanasiou A, Michalinos A, Alexandrou A, Georgopoulos S, Felekouras E. Inferior mesenteric arteriovenous fistula: case report and world-literature review. World J Gastroenterol. Jul 7 2014;20(25):8298-303. doi:10.3748/wjg.v20.i25.8298

8. Bronswijk M, De Hertogh G, Ferrante M. Edematous Colitis of an Unknown Cause, Diagnosis by Pattern Recognition. Gastroenterology. Jan 2019;156(1):e12-e13. doi:10.1053/j.gastro.2018.08.055

\section{Figure 1}
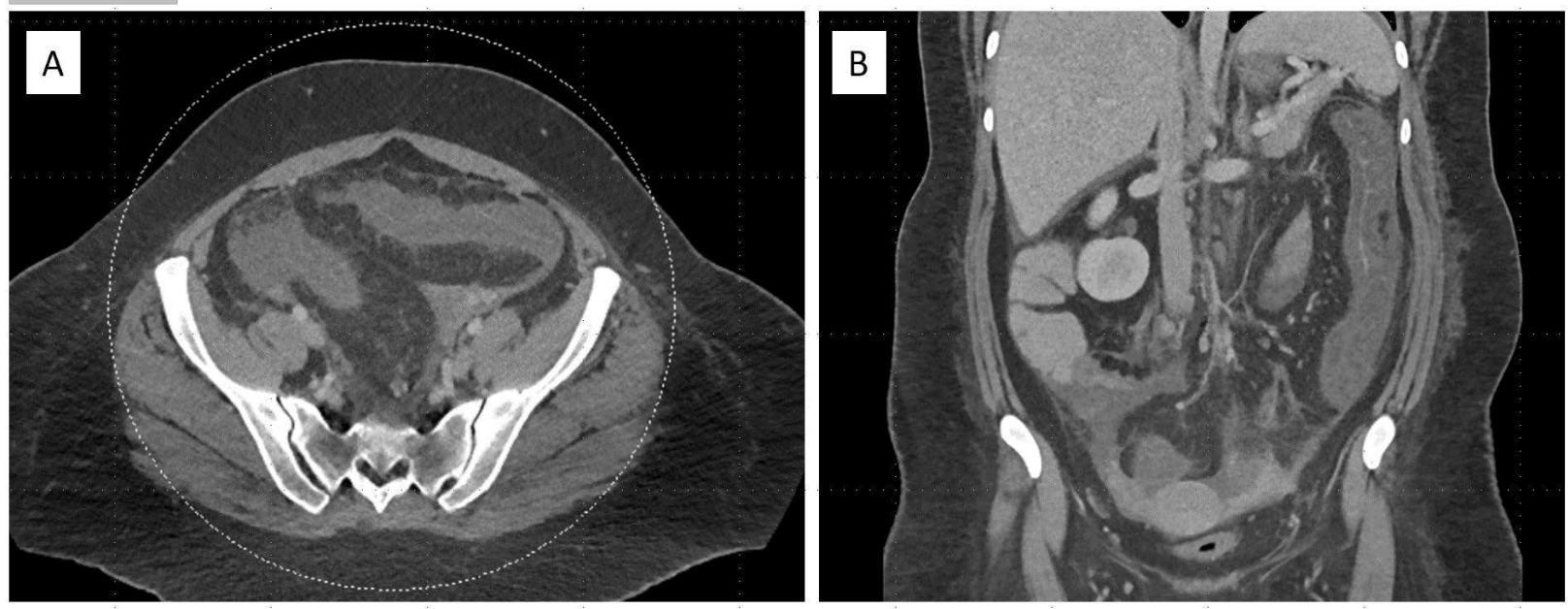

Axial (A) and Sagittal (B) venous phase CT imaging of the abdomen and pelvis showing colonic wall thickening and pericolonic inflammatory changes extending from the splenic flexure to the rectum. 


\section{Figure 2}
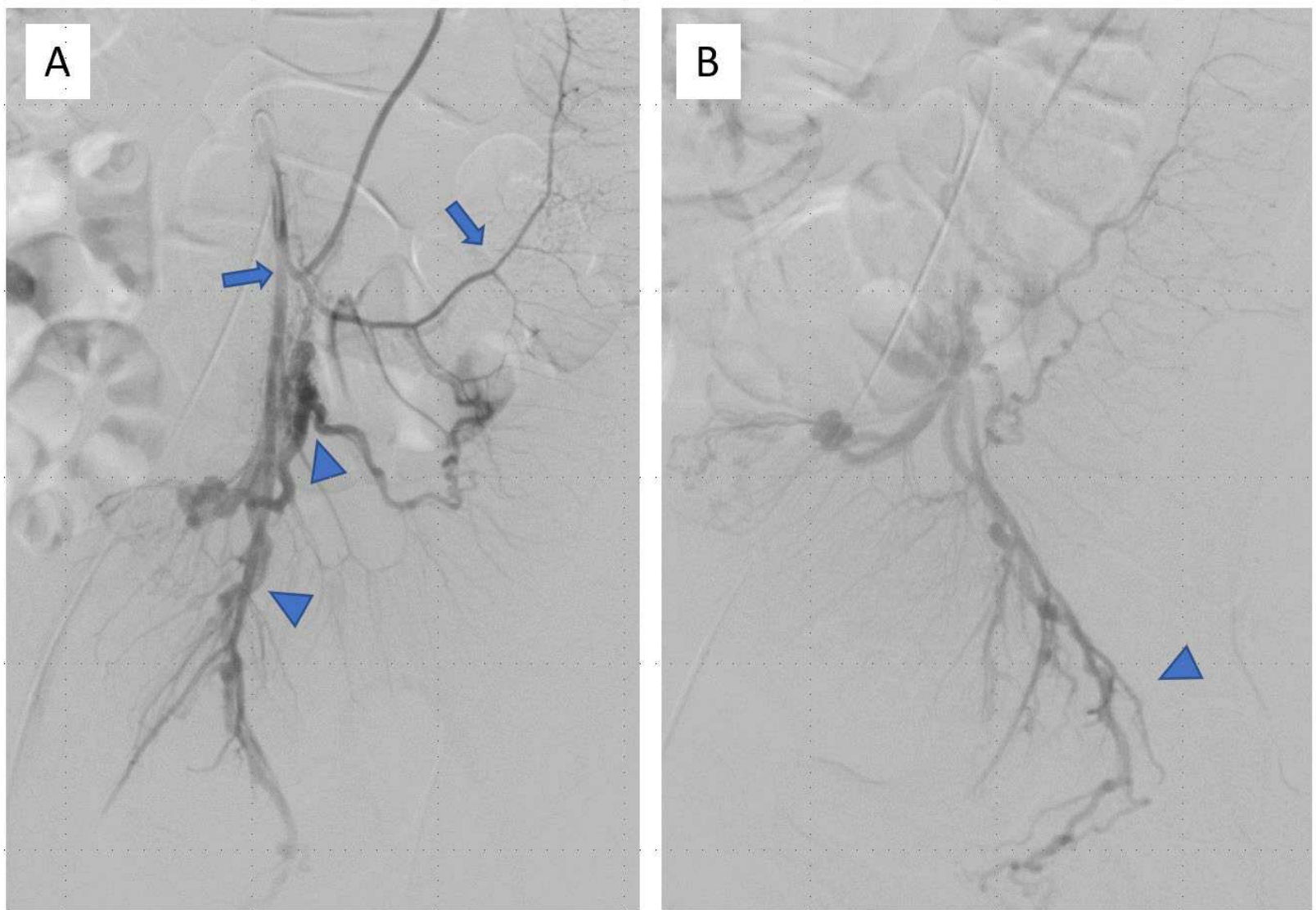

(A) Digital subtraction angiography of the inferior mesenteric artery shows simultaneous filling of the arterial system (arrow) and the venous mesenteric system (arrowhead). (B) Delayed imaging shows filling of the rectal venous plexus (arrowhead). 


\section{Figure 3}

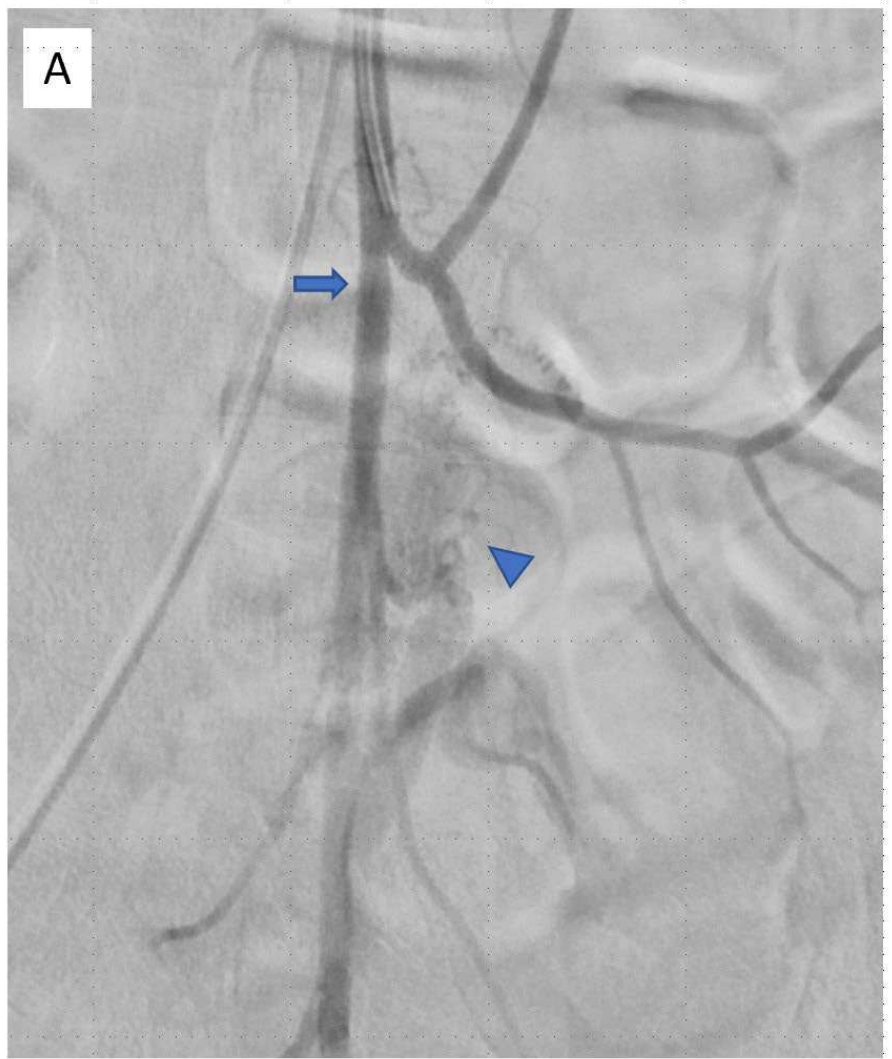

B

(A) Digital subtraction angiography of the inferior mesenteric artery through a 5 French catheter filling of the IMA (arrow) and filling of a nidus (arrowhead). (B) Superselective catheterization of a distal IMA branch with a microcatheter shows predominant filling of the nidus (arrowhead) and downstream filling of venous vasculature (arrow). Glue embolization was performed at this location. 


\section{Figure 4}

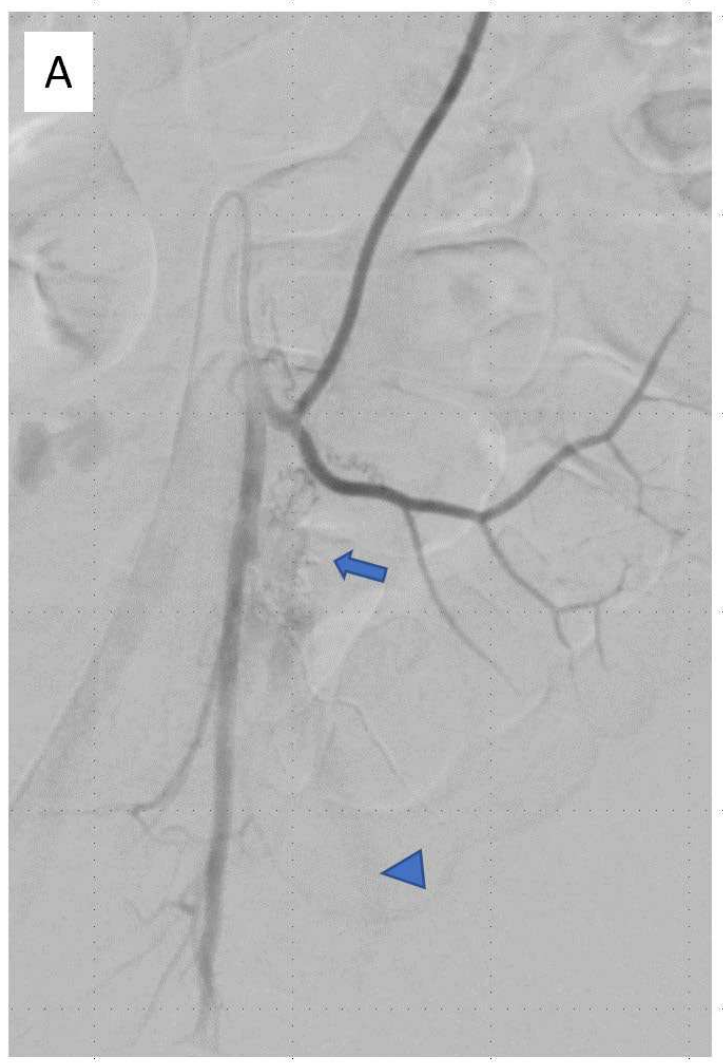

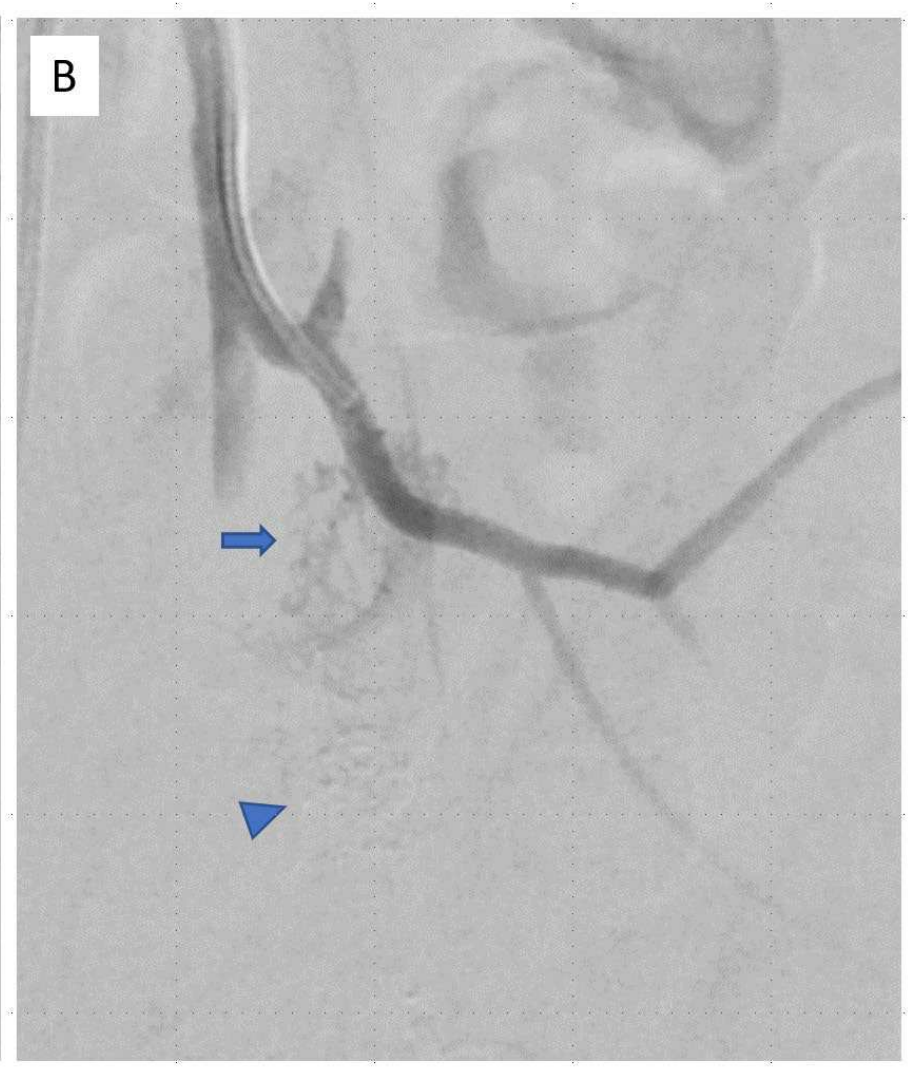

(A) Post-embolization digital subtraction angiography of the inferior mesenteric artery through a 5 French catheter filling of the IMA shows decreased but persistent filling of the nidus (arrow). There is also persistent but delayed filling of the venous system (arrowhead). (B) Selective catheterization of a proximal IMA branch with DSA showing small feeding vessels. There is no filling of the embolized portion of the nidus (arrowhead). 


\section{Declarations}

\section{Ethics approval and consent to participate}

Not applicable

\section{Consent for publication}

Written informed consent was obtained from the patient for publication of this case report and any accompanying images.

\section{Availability of data and materials}

Data sharing is not applicable to this article as no datasets were generated or analyzed during the current study.

\section{Competing interests}

The authors declare that they have no competing interests.

\section{Funding}

Not applicable

\section{Authors' contributions}

YBS and DL performed literature review and wrote and revised the manuscript. TSK provided clinical expertise and oversaw the report. All authors read and approved the final manuscript. 